\title{
On the formation of hidden chaotic attractors and nested invariant tori in the Sprott A system
}

\author{
Marcelo Messias · Alisson C. Reinol
}

Received: 20 September 2016 / Accepted: 8 December 2016 / Published online: 21 December 2016

(C) Springer Science+Business Media Dordrecht 2016

\begin{abstract}
We consider the well-known Sprott A system, which depends on a single real parameter $a$ and, for $a=1$, was shown to present a hidden chaotic attractor. We study the formation of hidden chaotic attractors as well as the formation of nested invariant tori in this system, performing a bifurcation analysis by varying the parameter $a$. We prove that, for $a=0$, the Sprott A system has a line of equilibria in the $z$-axis, the phase space is foliated by concentric invariant spheres with two equilibrium points located at the south and north poles, and each one of these spheres is filled by heteroclinic orbits of south pole-north pole type. For $a \neq 0$, the spheres are no longer invariant algebraic surfaces and the heteroclinic orbits are destroyed. We do a detailed numerical study for $a>0$ small, showing that small nested invariant tori and a limit set, which encompasses these tori and is the $\alpha$ - and $\omega$-limit set of almost all orbits in the phase space, are formed in a neighborhood of the origin. As the parameter $a$ increases, this limit set evolves into a hidden chaotic attractor, which
\end{abstract}

\section{Messias ( $\square)$}

Departamento de Matemática e Computação, Faculdade de Ciências e Tecnologia - FCT, UNESP Univ Estadual Paulista, Presidente Prudente, SP, Brasil e-mail: marcelo@fct.unesp.br

\section{A. C. Reinol}

Departamento de Matemática, Instituto de Biociências, Letras e Ciências Exatas - IBILCE, UNESP Univ Estadual Paulista, São José do Rio Preto, SP, Brasil

e-mail: alissoncarv@gmail.com coexists with the nested invariant tori. In particular, we find hidden chaotic attractors for $a<1$. Furthermore, we make a global analysis of Sprott A system, including the dynamics at infinity via the Poincaré compactification, showing that for $a>0$, the only orbit which escapes to infinity is the one contained in the $z$-axis and all other orbits are either homoclinic to a limit set (or to a hidden chaotic attractor, depending on the value of $a$ ), or contained on an invariant torus, depending on the initial condition considered.

Keywords Sprott A system · Hidden chaotic attractor . Invariant algebraic surfaces - Nested invariant tori · Homoclinic and heteroclinic orbits

\section{Introduction}

Chaotic systems have been intensively studied since Lorenz found, in 1963, the first chaotic attractor in a three-dimensional autonomous system [17,27], and various chaotic systems have been reported in the last years, as the Rössler system [24], the Chen system [1], the Lü system [18], and many others.

Recently, there is a special interest in finding and studying chaotic systems with hidden attractors, which are attractors whose basin of attraction does not intersect with small neighborhoods of any equilibrium point; for more details about this kind of attractors, see $[4,12]$ and references therein. Chaotic attractors in differential systems without any equilibrium point $[8,29]$, 
with only stable equilibria $[10,11,20,30-33]$ or with an infinite number of equilibrium points $[5,7,14]$, are hidden attractors. Here we call this kind of attractors hidden chaotic attractors. Systems with hidden chaotic attractors are rarely found; then, only a few examples have been reported in the literature, and there is little knowledge about their formation, and also an analytic proof of their existence is yet needed. In fact, while the classical chaotic attractors, as the Lorenz and Chen attractors, have some known routes to their formation, as the bifurcation of homoclinic orbits (Shilnikov-like theorems) or cascade of period doubling bifurcations, as far as we know very little is reported in the literature about the formation of hidden chaotic attractors. From the point of view of applications, hidden attractors are important in engineering applications because they allow unexpected and potentially disastrous responses to perturbation in a structure like a bridge or an airplane wing, as cited in $[7,9,20]$. Other recent applications and implementations of differential systems having hidden attractors can be found in [3,13,21,22,26,34].

In this paper, we consider the oldest and best-known example of differential system with a hidden chaotic attractor, given by

$$
\dot{x}=y, \quad \dot{y}=-x-y z, \quad \dot{z}=y^{2}-a,
$$

where $a \in \mathbb{R}$ and the dot denotes derivative with respect to the independent variable $t$, usually called the time. System (1) is a special case of the Nosé-Hoover oscillator [6] which describes many natural phenomena, as shown in [23], having practical as well as theoretical importance. In [28], Sprott listed system (1) as one of the differential systems without equilibrium points presenting chaotic behavior (case A), when $a=1$. In this way, system (1) is usually called Sprott A system. In [9], the authors showed that there exist nested invariant tori in the phase space of system (1) coexisting with the hidden chaotic attractor found in [28].

Aiming to understand how hidden chaotic attractors and invariant tori are formed in the Sprott A system, we perform a bifurcation analysis of system (1) by varying the parameter $a$. We prove that for $a=0$, the spheres $x^{2}+y^{2}+z^{2}=r^{2}$, with $r>0$, are invariant by the flow of system (1), so we call them invariant spheres. Hence, the phase space of system (1) with $a=0$ is foliated by invariant spheres and we prove that each of them is filled by heteroclinic orbits of south pole-north pole type. More precisely, the following result holds.
Theorem 1 For $a=0$, the phase space of system (1) is foliated by the invariant spheres $x^{2}+y^{2}+z^{2}=r^{2}$, with $r>0$, and the $z$-axis is a line of equilibria. Hence, the south pole $P_{-}$and the north pole $P_{+}$of each invariant sphere are equilibrium points of system (1) and the following statements hold.

(i) If $0<r<2$, then $P_{-}$is an unstable focus and $P_{+}$is a stable focus;

(ii) If $r=2$, then $P_{-}$is an unstable improper node and $P_{+}$is a stable improper node;

(iii) If $r>2$, then $P_{-}$is an unstable node and $P_{+}$is a stable node.

Furthermore, $P_{-}$and $P_{+}$are equilibrium points normally hyperbolic to the z-axis and for any orbit (different for $P_{-}$and $P_{+}$) on any invariant sphere of system (1), the $\alpha$-limit set is the unstable equilibrium point $P_{-}$ and the $\omega$-limit set is the stable equilibrium point $P_{+}$. Therefore, each invariant sphere is filled by an infinite set of heteroclinic orbits of south pole-north pole type, as shown in Fig. 1.

Theorem 1 is proved in Sect. 2 and describes the existence of a compact structure of the orbits in the phase space of system (1) with $a=0$, determined by the existence of the concentric invariant spheres. This compact structure plays an important role in the formation of hidden chaotic attractors and invariant tori, for $a>0$ small enough. In fact when $a \neq 0$, the heteroclinic orbits described in Theorem 1 are destroyed, since system (1) has no equilibrium points for $a \neq 0$, and the spheres $x^{2}+y^{2}+z^{2}=r^{2}$ are no longer invariant algebraic surfaces. Furthermore, the $z$-axis becomes invariant under the flow of system (1). Based on these observations, we study the dynamical consequences of these bifurcations in the phase space of system (1) by varying the parameter $a$; in particular, we relate them to the formation of hidden chaotic attractors and invariant tori. We performed a detailed numerical analysis of system (1) for $a>0$ small. The main results obtained are summarized in the following numerical result and are presented with details in Sect. 3.

Numerical Result 2 For $a>0$ small enough, there exist small nested invariant tori in a neighborhood of the origin in the phase space of system (1), as shown in Fig. 2a, and the structure of invariant spheres is preserved, unless in a tubular neighborhood of the invariant z-axis, as shown in Fig. 3. The orbits with initial condition on these spherical structure are homoclinic 

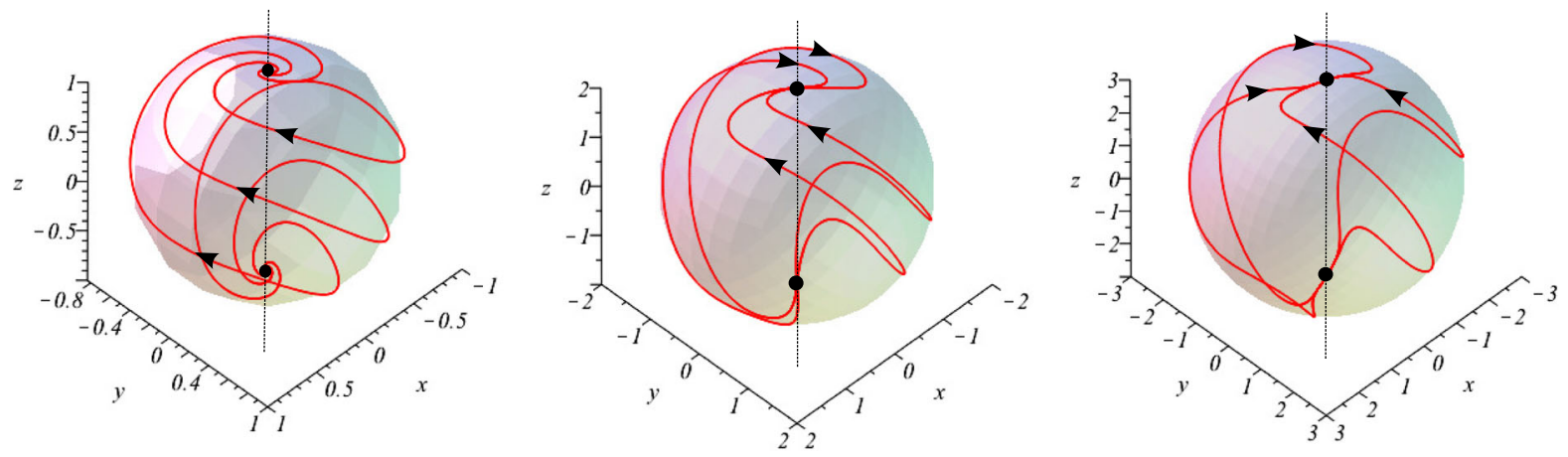

$$
0<r<2
$$

$$
r=2
$$$$
r>2
$$

Fig. 1 Flow of system (1) restricted to the invariant spheres $x^{2}+y^{2}+z^{2}=r^{2}$ for $0<r<2, r=2$ and $r>2$

Fig. 2 a Small nested invariant tori of Sprott A system, obtained taking initial conditions

$(0,0.01,0)($ red $)$ and $(0,0.025,0)$ (gray). b Orbit with initial condition $(0,1,0)$ (blue) homoclinic to a limit set $(\mathrm{red})$. In both cases, we take system (1) with $a=10^{-4}$. (Color figure online)

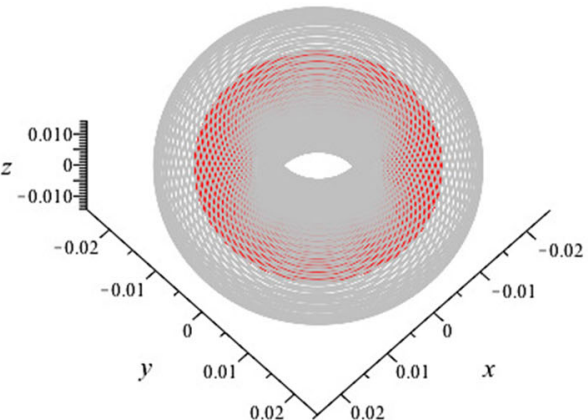

(a)

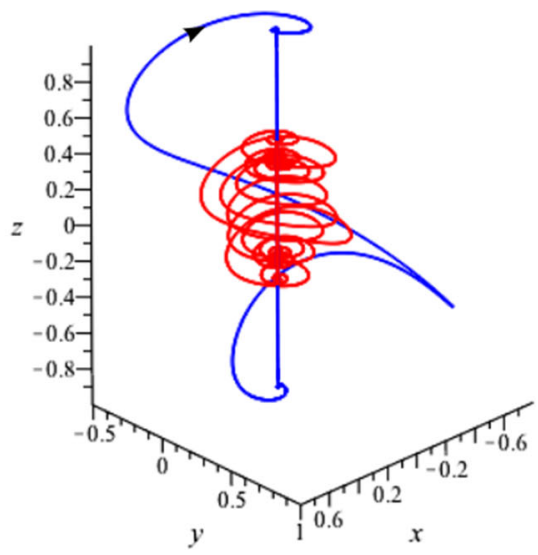

(b) to a "limit set" which is formed in a neighborhood of the origin, as drawn in Fig. 2b. This limit set encompasses the small nested invariant tori. Furthermore, as the parameter a increases, the invariant tori and the limit set expand and the limit set evolves into a hidden chaotic attractor of system (1), as illustrated in Fig. 4. The hidden chaotic attractor coexists with the nested invariant tori, yet encompassing them (see Fig. 13 in Sect. 3).

Here we say that an orbit is homoclinic to a limit set when there exists a set which is the $\alpha$ - and the $\omega$ limit sets of the orbit, as the one described in Numerical Result 2 and shown in Fig. 2 b. Observe that the union of these orbits with initial conditions on the same sphere $x^{2}+y^{2}+z^{2}=r^{2}$ forms a structure as the one drawn in Fig. 5 which resembles an "apple" (see also Fig. 3a). In Fig. 4 is shown the orbit with initial condition $(0,5,0)$ for different values of the parameter $a$, that is, $a=$ $10^{-4}, a=10^{-2}, a=0.1, a=0.25$, and $a=0.4$. Note that for each $a>0$ sufficiently small, this orbit is homoclinic to a specific limit set. As the parameter $a$ increases, the limit set expands, and for $a=0.4$, it can be characterized as a hidden chaotic attractor, as shown in Sect. 3 by calculating the Lyapunov exponents and the Lyapunov dimension of this attractor. Hence, we detected a hidden chaotic attractor for Sprott A system, coexisting with nested invariant tori, even for $a<1$.

Also, the numerical results obtained suggest that for each small enough value of the parameter $a$, almost all orbits in the phase space of system (1), except the ones contained in the $z$-axis and in the nested invariant tori, have a set which is their $\alpha$ - and $\omega$-limit sets. This set may be a hidden chaotic attractor, depending on the parameter value. Hence, in order to confirm these assertions and better understand the dynamics of system (1), 


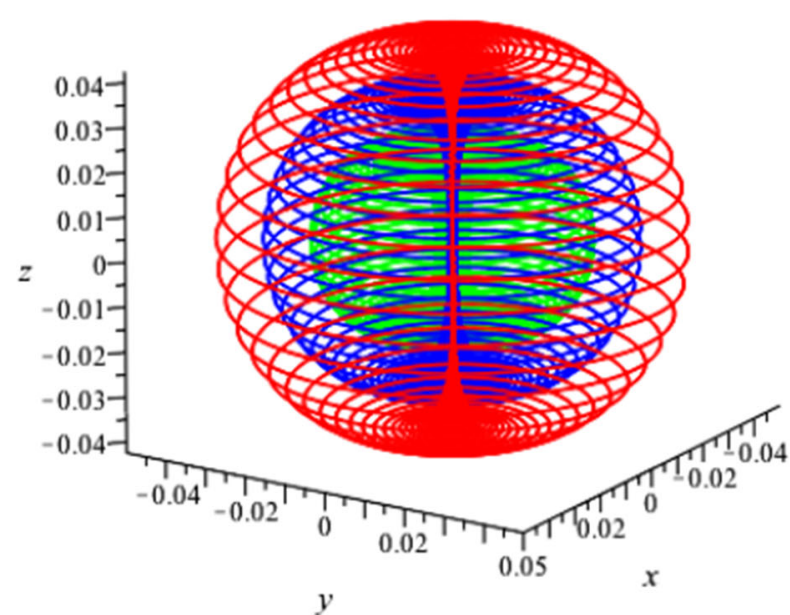

(a)

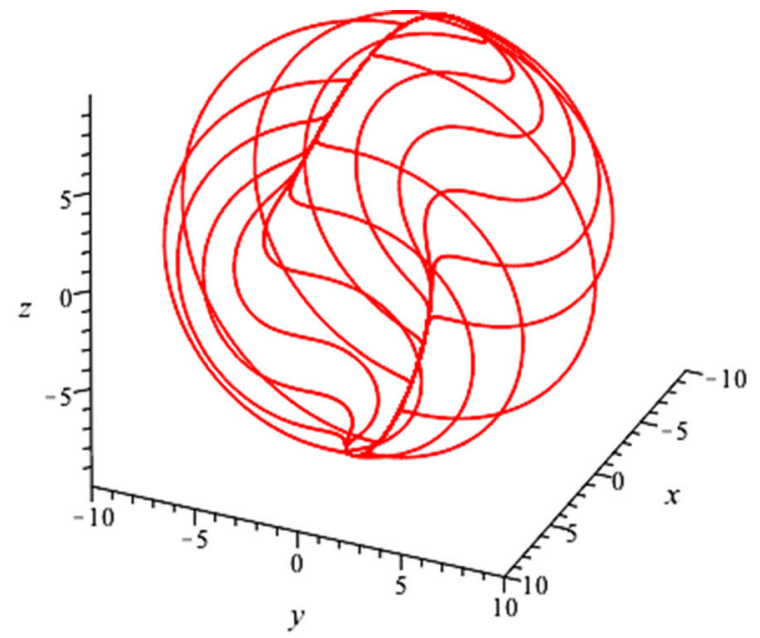

(b)

Fig. 3 a Orbits with initial conditions on the spheres of radius $r=0.03$ (green), $r=0.04$ (blue), and $r=0.05$ (red). b Several orbits with initial conditions on the sphere of radius $r=10$. In both cases, $a=10^{-4}$. The structure of the invariant spheres, for

we perform a global analysis of its orbits. By using the Poincaré compactification, we study the dynamics of system (1) on the sphere at infinity and in a neighborhood of this sphere. The obtained results are described in the next theorem.

Theorem 3 For all values of the parameter $a \in \mathbb{R}$, the phase portrait of system (1) on the Poincaré sphere $\mathbb{S}^{2}$ (at infinity) is as shown in Fig. 6: There exist a great circle of equilibria and an infinite set of heteroclinic orbits connecting pairs of equilibrium points of this circle. Furthermore, the only orbit of system (1) which escapes to infinity is the one contained in the invariant $z$-axis, and there exist orbits with large amplitude, which are homoclinic to the limit set described in Numerical Result 2.

In Sect. 4, we prove Theorem 3. Note that in the Poincaré sphere $\mathbb{S}^{2}$ (at infinity), there is an infinite set of heteroclinic orbits, which exists for all values of the parameter $a \in \mathbb{R}$ and, consequently, even when system (1) has chaotic behavior.

In the rest of this paper, we prove Theorems 1 and 3 and present the computational analysis summarized in Numerical Result 2 and in the observations given in this introduction. In this way, we intend to give a contribution to the understanding of the complex dynamical behavior of the Sprott A system, in particular on the formation of hidden chaotic attractors and invariant tori.

"smal" radius a and "big" radius $\mathbf{b}$, is preserved by continuity, unless in a tubular neighborhood of the invariant $z$-axis. (Color figure online)

\section{Existence of a compact structure and the proof of Theorem 1}

Consider $a=0$ into the Sprott A system (1). In this case, system (1) has a line of equilibria given by the $z$-axis. Moreover, it is easy to check that the analytic function $f(x, y, z)=x^{2}+y^{2}+z^{2}$ is a first integral of system (1), since $\langle X, \nabla f\rangle=0$, where $X=\left(y,-x-y z, y^{2}\right)$ is the vector field associated with system (1) with $a=0$. Hence, the spheres $x^{2}+y^{2}+z^{2}=r^{2}$, with $r>0$, are invariant by the flow of system (1).

The intersection points of the $z$-axis with the invariant spheres $x^{2}+y^{2}+z^{2}=r^{2}$ are $P_{-}=(0,0,-r)$ and $P_{+}=(0,0, r)$, that is the south pole and the north pole, respectively, of each invariant sphere.

For each invariant sphere $x^{2}+y^{2}+z^{2}=r^{2}$, the eigenvalues of the linear part of system (1) at the equilibrium points $P_{ \pm}$are

$\lambda_{1}=\mp \frac{1}{2} r+\frac{1}{2} \sqrt{r^{2}-4}$,

$\lambda_{2}=\mp \frac{1}{2} r-\frac{1}{2} \sqrt{r^{2}-4}, \quad \lambda_{3}=0$,

with corresponding eigenvectors

$v_{1}=\left(\frac{2}{\mp r+\sqrt{r^{2}-4}}, 1,0\right)$, 


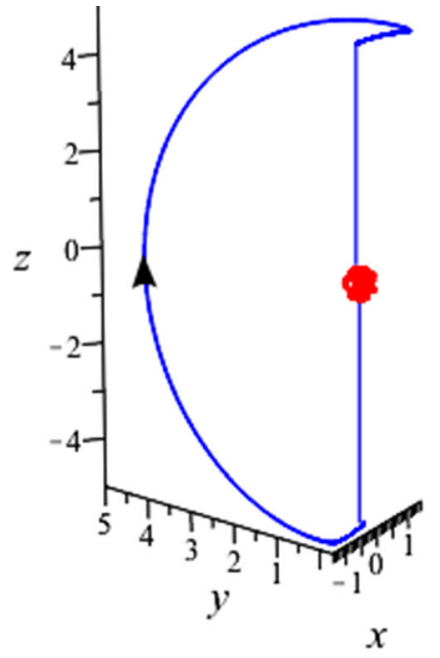

(a)

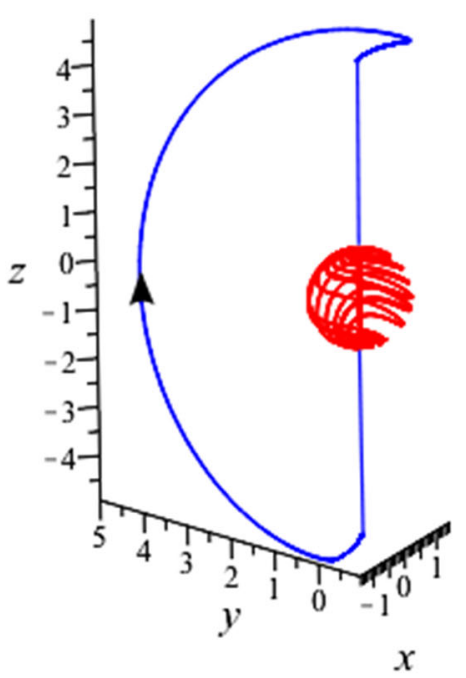

(b)

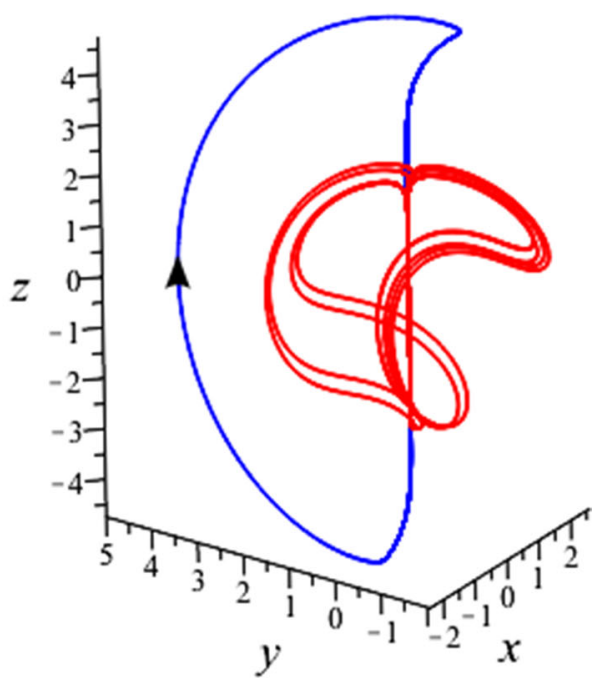

(c)

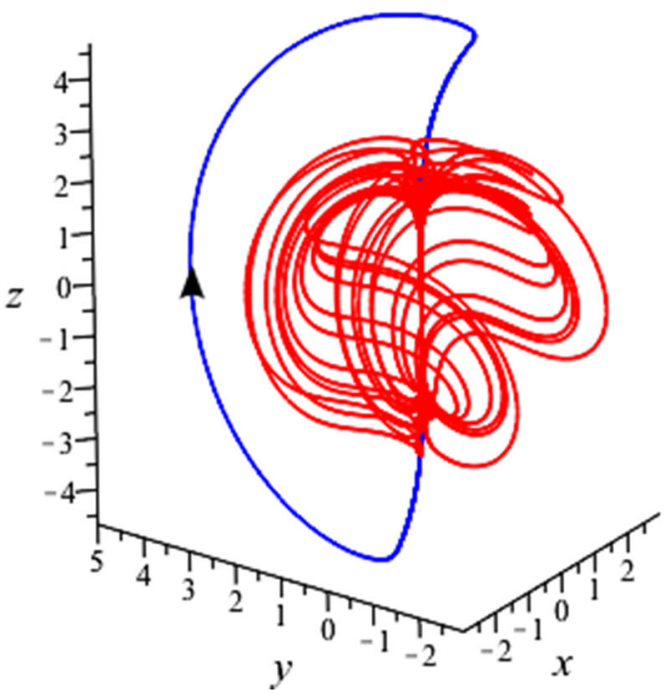

(d)

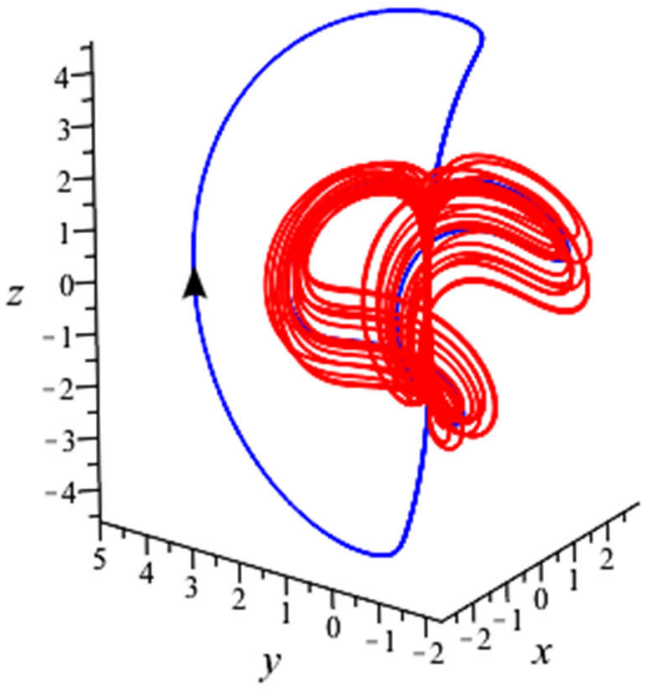

(e)

Fig. 4 Orbit with initial condition $(0,5,0)$ for: $\mathbf{a} a=10^{-4} ; \mathbf{b} a=10^{-2} ; \mathbf{c} a=0.1 ; \mathbf{d} a=0.25$; and e $a=0.4$. The limit set (red) evolves into a hidden chaotic attractor. (Color figure online)

$v_{2}=\left(\frac{2}{\mp r-\sqrt{r^{2}-4}}, 1,0\right), \quad v_{3}=(0,0,1)$.

We have the following cases to consider:

1. If $0<r<2$, then the eigenvalues $\lambda_{1,2}$ are complex with positive real part for $P_{-}$and negative real part for $P_{+}$. Hence, the equilibrium point $P_{-}$is an unstable focus and the equilibrium point $P_{+}$is a stable focus. Considering also the corresponding eigenvectors, it is easy to see that the orbits locally spiraling toward the equilibrium point $P_{+}$, when $t \rightarrow+\infty$, on a surface tangent to the plane spanned by the eigenvectors $v_{1,2}$, hence in a direction normal to the $z$-axis. The same is true for the equilibrium point $P_{-}$when $t \rightarrow-\infty$ (see Fig. 1, left). 


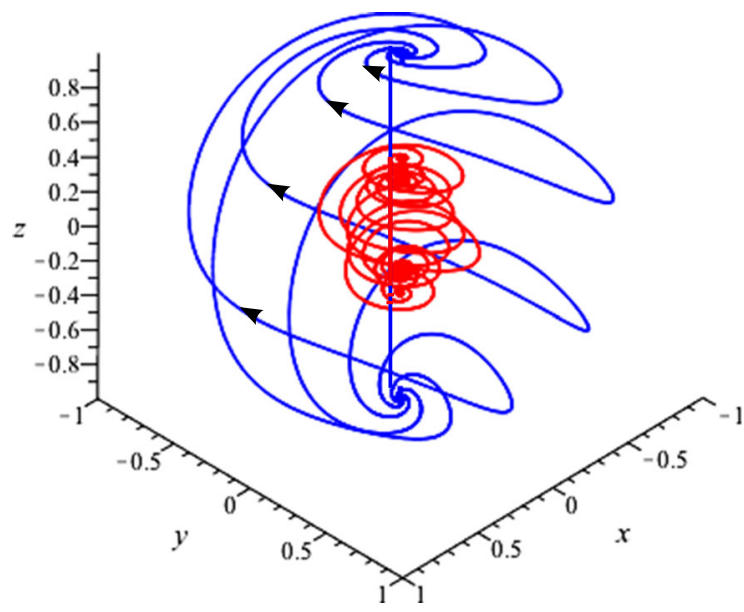

Fig. 5 "Apple" structure formed by orbits homoclinic to a limit set and with initial conditions on the sphere $x^{2}+y^{2}+z^{2}=1$, for $a=10^{-4}$

2. If $r=2$, then the eigenvalues $\lambda_{1,2}$ are real and $\lambda_{1}=\lambda_{2}>0$ for $P_{-}$and $\lambda_{1}=\lambda_{2}<0$ for $P_{+}$. Hence, $P_{-}$is an unstable improper node and $P_{+}$is a stable improper node. Considering the eigenvectors $v_{1,2}$, we can conclude that $P_{-}$and $P_{+}$are nodes normally hyperbolic to the $z$-axis (see Fig. 1, central).

3 . If $r>2$, then the eigenvalues $\lambda_{1,2}$ are real and positive for $P_{-}$and real and negative for $P_{+}$. Hence, $P_{-}$is an unstable node and $P_{+}$is a stable node. Taking into account the eigenvectors $v_{1,2}$, we have that the nodes $P_{-}$and $P_{+}$are normally hyperbolic to the $z$-axis (see Fig. 1, right).

Observe that the origin is a degenerate equilibrium point of system (1) and the behavior of the orbits in a neighborhood of it is determined considering the orbits of case 1 when $r \rightarrow 0$.

The type and stability of the equilibrium points $P_{ \pm}$ described above are only local. So, in order to prove that each invariant sphere of system (1) is filled by heteroclinic orbits of south pole-north pole type, we should study the flow restricted to these spheres. For this purpose, we consider the following local charts.

Assume that $z>0$. Taking $z=\sqrt{r^{2}-x^{2}-y^{2}}$ into system (1), we obtain the planar differential system

$\dot{x}=y, \quad \dot{y}=-x-y \sqrt{r^{2}-x^{2}-y^{2}}$.

The origin is the only equilibrium point of system (2), and the eigenvalues of the linear part of this system at this point are

$\lambda_{1,2}=-\frac{1}{2} r \pm \frac{1}{2} \sqrt{r^{2}-4}$.

Hence, the origin is a stable focus if $0<r<2$, a stable improper node if $r=2$ and a stable node if $r>2$. Analogously, when $z<0$ we take $z=-\sqrt{r^{2}-x^{2}-y^{2}}$ into system (1) and the only equilibrium point of the obtained system is the origin which is an unstable focus if $0<r<2$, an unstable improper node if $r=2$ and an unstable node if $r>2$. In Fig. 7 are drawn the phase portraits of system (1) in the local charts $z>0$ (up) and $z<0$ (below) when $0<r<2, r=2$ and $r>2$.

We claim that there is no periodic orbits of system (1) on the invariant spheres. Indeed, considering
Fig. 6 Phase portrait of system (1) on the sphere at infinity: great circle of equilibria (red) and heteroclinic orbits connecting pairs of these equilibria. (Color figure online)

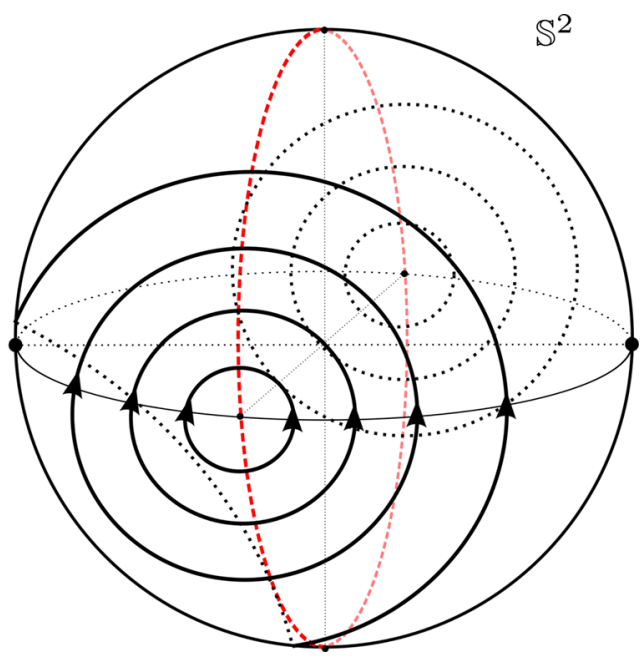



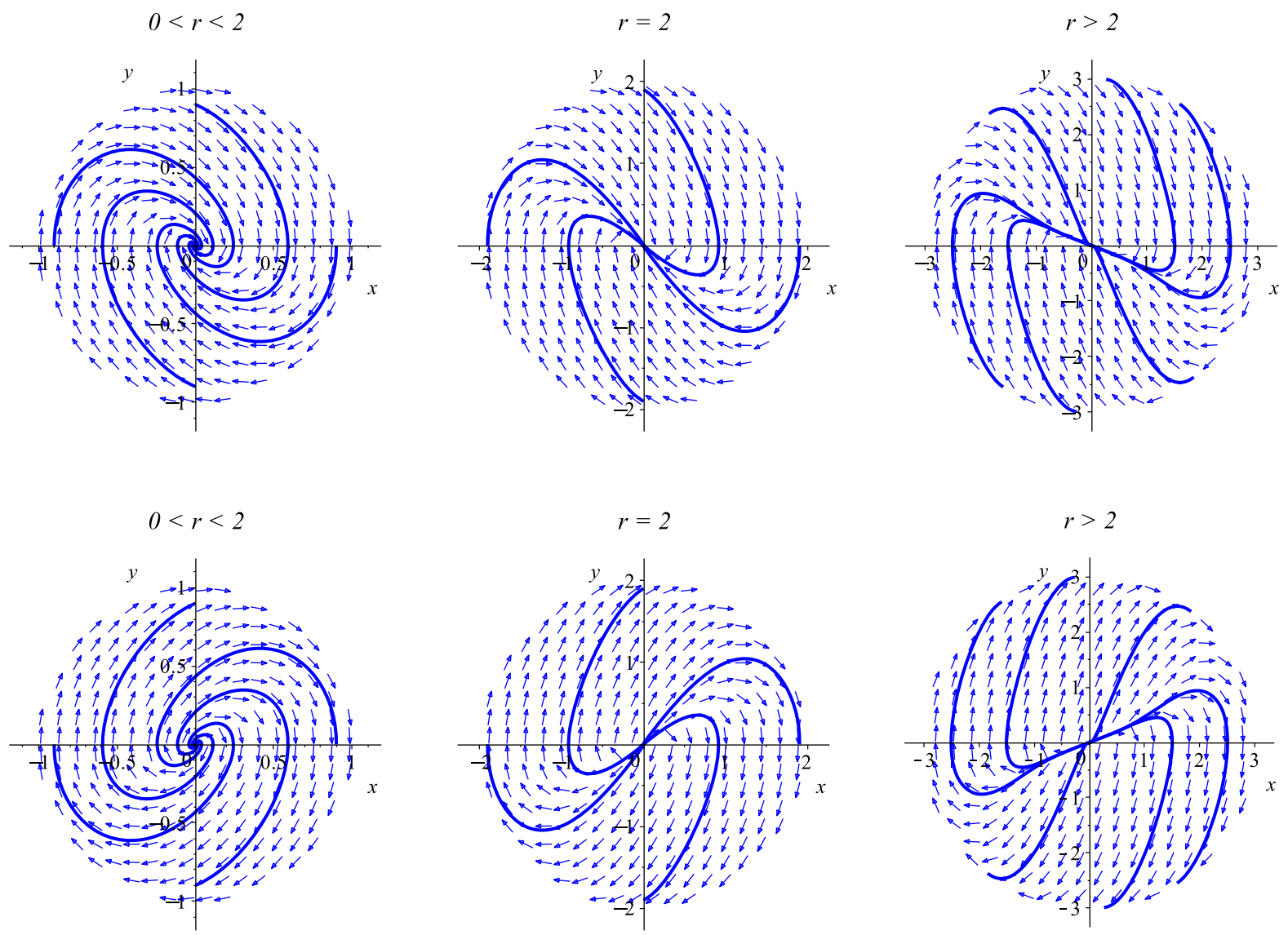

Fig. 7 Phase portraits of system (1) restricted to the invariant spheres, on the local charts $z>0$ (up) and $z<0$ (below), for $0<r<2$, $r=2$ and $r>2$

the flow in the local charts $z>0$ and $z<0$ studied above, the only possibility for the existence of a periodic orbit is on the equator of the invariant spheres. However, $\dot{z}>0$ for $z=0$. Then, the flow of system (1) on the equator of each invariant sphere is increasing; hence, there is no periodic orbit contained there.

As on each invariant sphere, the south pole $P_{-}$and the north pole $P_{+}$are the only equilibrium points and there is no periodic orbits, by the Poincaré-Bendixson Theorem, the $\alpha$-limit set of all orbits on each invariant sphere is the unstable equilibrium point $P_{-}$and the $\omega$ limit set of them is the stable equilibrium point $P_{+}$. In other words, all orbits on each invariant sphere (except $P_{-}$and $P_{+}$) tend toward the south pole $P_{-}$when $t \rightarrow$ $-\infty$ and they tend toward the north pole $P_{+}$when $t \rightarrow$ $+\infty$, forming an infinite set of heteroclinic orbits of south pole-north pole type. This proves Theorem 1 .

\section{Numerical study of Sprott A system for $\boldsymbol{a}>0$ small}

In [28], Sprott found a hidden chaotic attractor for system (1) with $a=1$ and taking the initial condition $(0,5,0)$. Based on the results obtained in the previous section, one can say that the existence of a compact structure in the phase space of system (1) with $a=0$, given by the existence of invariant spheres and heteroclinic orbits contained on them, plays an important role in the formation of hidden chaotic attractors and invariant tori. Thus, in this section we perform a detailed numerical study of system (1) for $a>0$ small, when the spheres are no longer invariant algebraic surfaces and the heteroclinic orbits are destroyed, describing the dynamical consequences of these bifurcations and relating them to the formation of hidden chaotic attractors and invariant tori. 
Fig. 8 Orbit with initial condition $(0.001,0,0.5)$ when $a=10^{-6}$ and for the time $t$ negative (left) and positive (right)

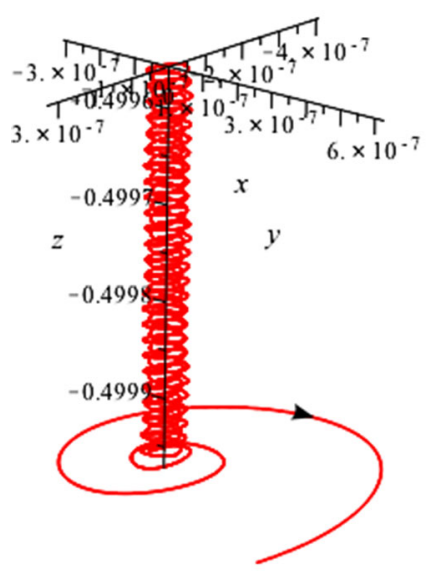

\subsection{Persistence of the compact structure}

As we proved in Sect. 2, when $a=0$ the $z$-axis is a line of equilibria of system (1) and there are infinitely many heteroclinic orbits of south pole-north pole type contained on invariant spheres. For $a>0$, system (1) has no equilibrium points and the $z$-axis becomes invariant by the flow of this system. Indeed, if $x=y=0$, then $\dot{z}=-a<0$. Hence, the orbits with initial condition on the $z$-axis are entirely contained there and they move toward its negative direction. However, for $a$ small enough, the compact structure of the phase space given by the invariant spheres is preserved, far from a tubular neighborhood of the $z$-axis, in the following sense: given a point in $\mathbb{R}^{3}$ far from the $z$-axis, it belongs to one of the spheres $x^{2}+y^{2}+z^{2}=r^{2}, \quad r>0$, which, by continuity, preserve its invariance with a small deformation, outside a tubular neighborhood of the $z$-axis, where there are no equilibrium points. The orbit passing by this point turn around the deformed sphere until it reaches the tubular neighborhood of the $z$-axis, in the future and in the past time (see Fig. 3); then the orbits oscillate around the $z$-axis toward a neighborhood of the origin, for negative and for positive times, as shown in Fig. 8. This dynamical behavior of the orbits leads to the formation of invariant tori and orbits homoclinic to a limit set (or to a hidden chaotic attractor), as we describe in the next subsection.
3.2 Formation of nested invariant tori around the origin

Consider a small invariant sphere of system (1), which exists for $a=0$, and take an initial condition on it. The orbit passing by this initial condition tends, in the future and in the past, toward equilibrium points in the $z$-axis, forming an heteroclinic orbit (see Fig. 9 a). Now, taking $a>0$ small enough, consider the same initial condition. Following the orbit passing by this initial condition, taking into account the assertions of Sect. 3.1, observe that it turns around a (deformed) sphere and then oscillates around the $z$-axis toward the origin, in negative and in positive time, but now forming an invariant torus, as we can see in Fig. 9b, c. A detailed numerical analysis performed suggests that there are a family of small nested invariant tori around the origin in the phase space, and the most external one is unstable in the following sense: an orbit with initial condition in an external neighborhood of this most external torus tends to a limit set which encompasses the family of nested invariant tori. In synthesis, a set of small concentric spheres, existing for $a=0$, turn into a set of small nested invariant tori, for $a>0$ small enough. On the other hand, the orbits contained in bigger spheres, roughly speaking, become homoclinic to a limit set, as explained ahead. 


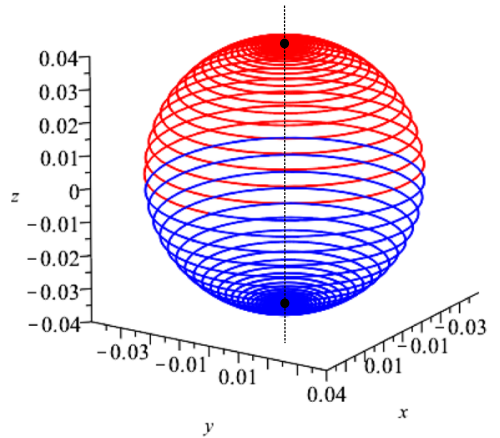

(a)

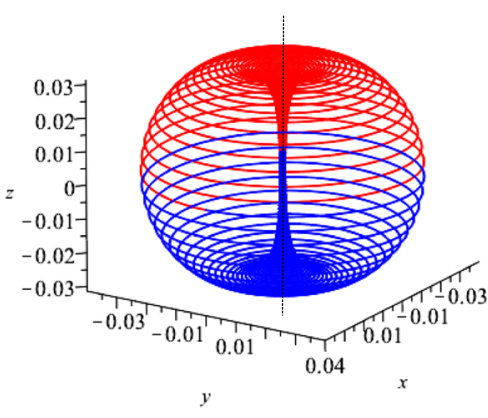

(b)

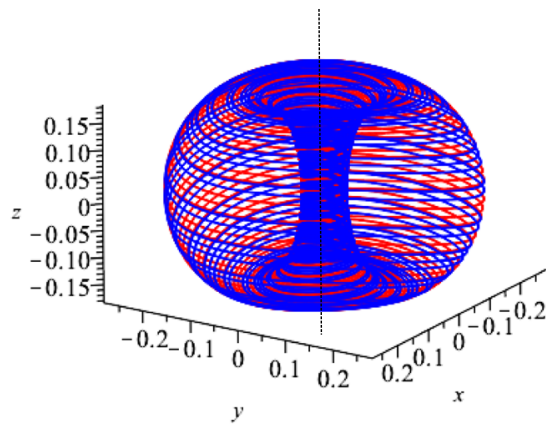

(c)

Fig. 9 Orbit with initial condition $(0.04,0,0)$ for $\mathbf{a} a=0, \mathbf{b} a=10^{-6}$ and $\mathbf{c} a=10^{-2}$ when the time $t$ is negative (blue) and positive (red). (Color figure online)

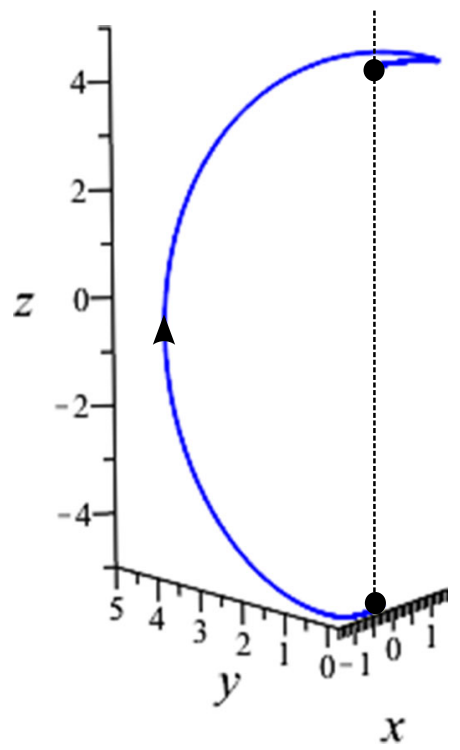

(a)

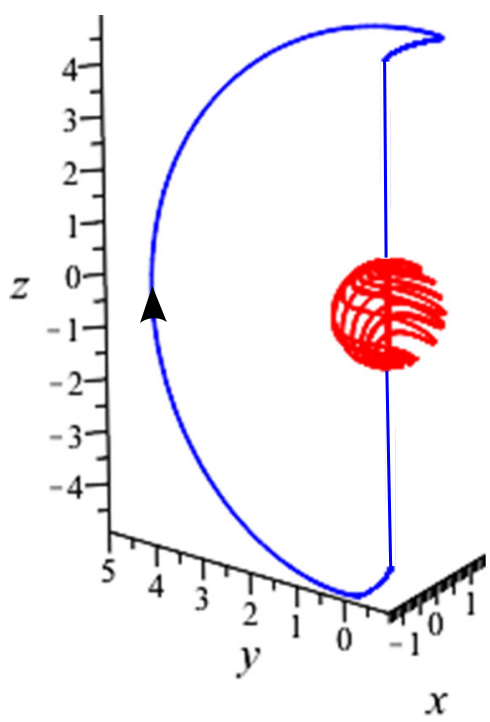

(b)

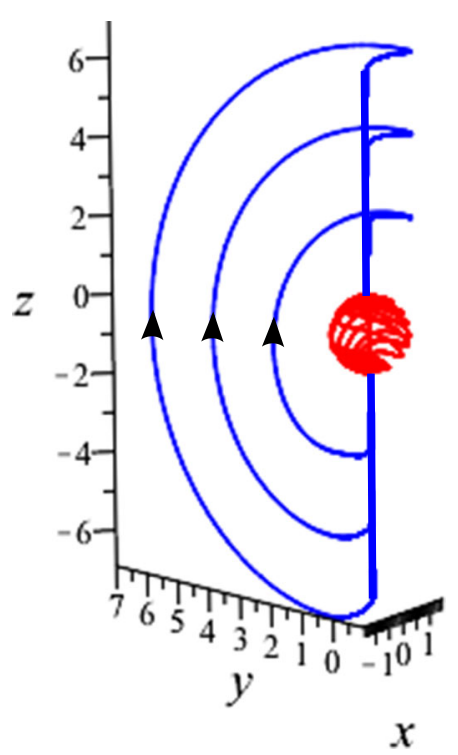

(c)

Fig. 10 Orbit with initial condition $(0,5,0)$ for $\mathbf{a} a=0$ and $\mathbf{b} a=10^{-2}$. c Orbits with initial conditions $(0,3,0),(0,5,0)$ and $(0,7,0)$ for $a=10^{-2}$

\subsection{Formation of a limit set}

Consider now a big invariant sphere which exists for $a=0$ and take an initial condition on it. Again, the orbit passing by this initial condition tends in the future and in the past toward equilibrium points in the $z$-axis, forming a heteroclinic orbit (see Fig. 10a). However, taking $a>0$ small enough, considering the same initial condition and following the orbit passing by this initial condition in the future and in the past, we observe that it turns around a (deformed) sphere and then oscillates around the $z$-axis toward the origin, tending to a limit set as $t \rightarrow \pm \infty$ (see Fig. 10b, where the limit set is drawn in red). The same happens for any sufficiently large sphere considered. In this way, we have numerical evidences that all the orbits in the phase space, unless the one contained in the $z$-axis and the ones on the nested invariant tori described in Sect. 3.2, are homoclinic to a limit set (which is fixed, for each fixed $a$ ), see Fig. 10c. We observe from the numerical analy- 


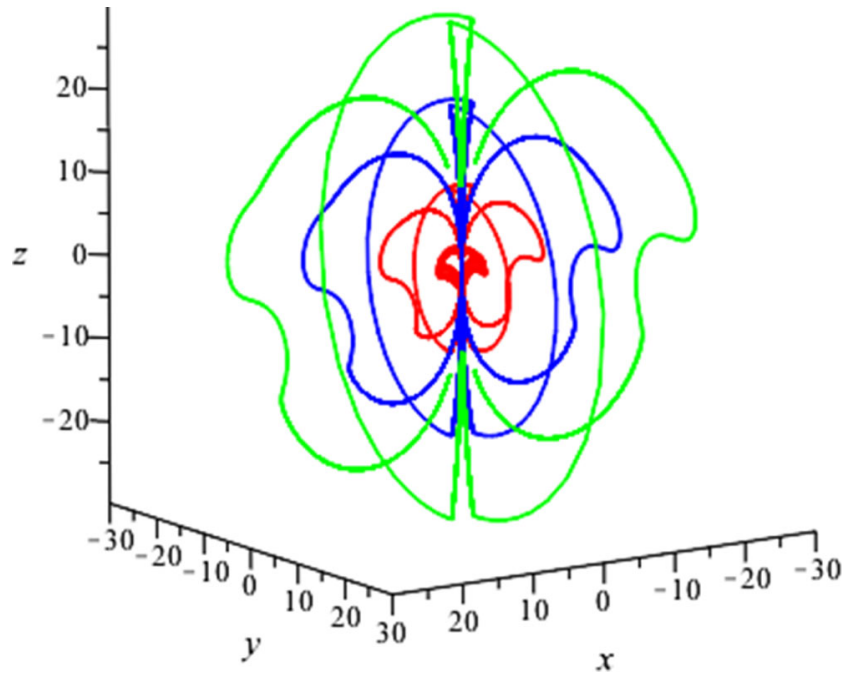

(a)

Fig. 11 a Orbits with initial conditions on the spheres of radius $r=10$ (red), $r=20$ (blue), and $r=30$ (green) for $a=0.4$. They are homoclinic to the hidden chaotic attractor. The compact

sis performed that this limit set encompasses the nested invariant tori described in Sect. 3.2. Moreover, the limit set evolves into a hidden chaotic attractor as the value of parameter $a$ increases, as we shall describe in the next subsection.

\subsection{Formation of a hidden chaotic attractor}

As the value of parameter $a$ increases, the nested invariant tori described in Sect. 3.2 and the limit set described in Sect. 3.3 expand. We observe that, by varying the parameter $a$, the limit set turn into a hidden chaotic attractor, as shown in Fig. 4, and the orbits become homoclinic to the hidden chaotic attractor, as shown in Figs. 4e and 11. Indeed, we find a hidden chaotic attractor for $a=0.4$, encompassing the nested invariant tori (see Fig. 13). In this way, we show that there exists a hidden chaotic attractor in the Sprott A system for $a<1$. In fact, the orbit with initial condition $(0,5,0)$, the same taken by Sprott in [28], is a heteroclinic orbit connecting two nodes when $a=0$, and, for $a>0$ small, it becomes an orbit homoclinic to a limit set. The bifurcation of this limit set as the parameter value $a$ increases is shown in Fig. 4, and for $a=0.4$, it is possible to observe the existence

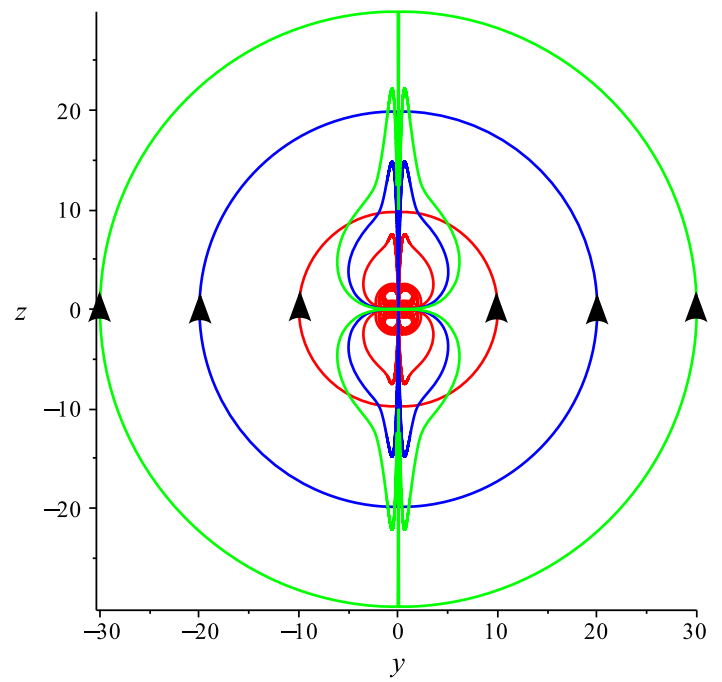

(b)

spherical structure is preserved by continuity, far from the $z$-axis, as shown in $\mathbf{b}$. (Color figure online)

of the hidden chaotic attractor, since $L E_{1}=0.0219$, $L E_{2} \approx 0, L E_{3}=-0.0219$ and $D_{L}=2.9908$, where $L E_{i}$ are the Lyapunov exponents and $D_{\mathrm{L}}$ is the Lyapunov dimension of this attractor. Here these quantities were calculated with the algorithm described in [25]. In Fig. 12 are drawn the $x$-coordinates of two solutions of system (1) for $a=0.4$, with initial conditions very close, given by $(0,5,0)$ and $(0,5.0001,0)$. Observe that they have an exponential divergence as the time $t$ increases, which confirms the chaotic behavior. We can also find chaotic behavior in system (1) to other parameter values of $a$ between 0.4 and 1, for example when $a=0.55$. Moreover, there are nested invariant tori in the phase space of system (1) with $a=0.4$, that is, even when it has chaotic behavior, as can be seen in Fig. 13. Other example of nested invariant tori coexisting with a hidden chaotic attractor in system (1) with $a=1$ was reported in [9] .

\section{Dynamics of system (1) at infinity: the proof of Theorem 3}

As any polynomial differential system, the Sprott A system (1) can be extended to an analytic system defined on a closed ball $\mathbb{D}^{3}$ of radius one, whose inte- 
Fig. 12 The $x$-coordinates of two solutions of the Sprott A system (1) for $a=0.4$, with initial conditions $(0,5,0)$ (red) and $(0,5.0001,0)$ (blue), showing the sensitive dependence on the initial conditions. (Color figure online)
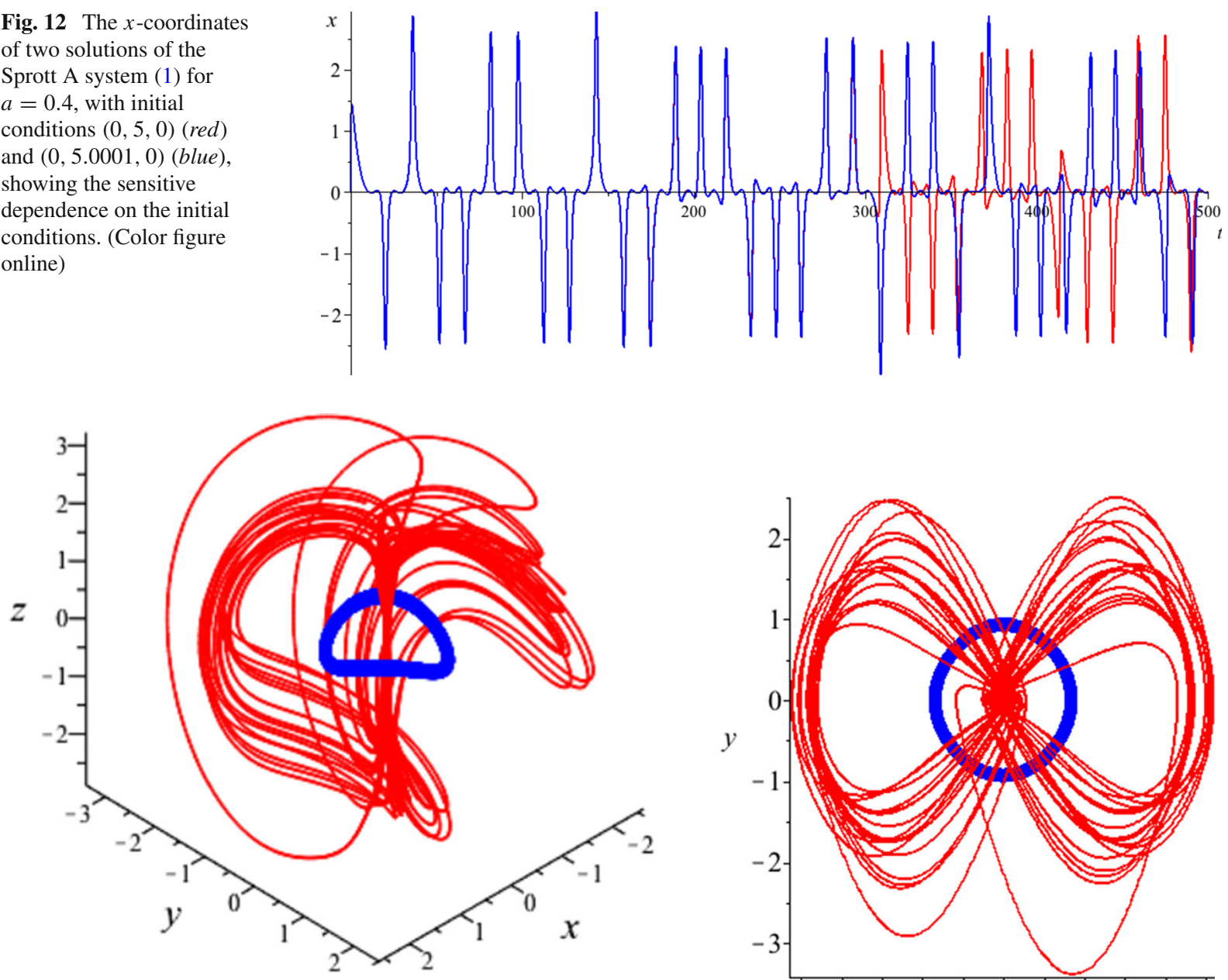

(a)

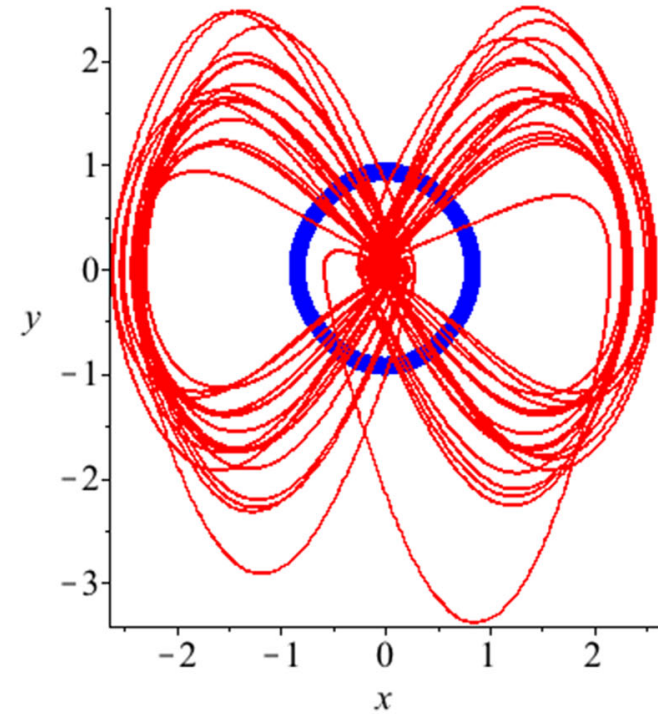

(b)

Fig. 13 a Orbits of the Sprott A system (1) with $a=0.4$ and initial conditions $(0,1,0)$ (blue - an invariant torus) and (0, 5, 0) (red hidden chaotic attractor). b Their projections on the plane- $x y$. (Color figure online)

rior is diffeomorphic to $\mathbb{R}^{3}$ and its invariant boundary, the two-dimensional sphere $\mathbb{S}^{2}=\{(x, y, z)$ : $\left.x^{2}+y^{2}+z^{2}=1\right\}$, plays the role of the infinity. This closed ball is known as Poincaré ball, since the technique for doing such an extension is precisely the Poincaré compactification for a polynomial differential system in $\mathbb{R}^{3}$, which is described in detail in [2]. A summary of this compactification technique and some applications of it can be found in $[15,16,19]$. When we perform the Poincaré compactification of system (1), we obtain six polynomial vector fields defined on the local charts $U_{i}$ and $V_{i}, i=1,2,3$, with coordinates $\left(z_{1}, z_{2}, z_{3}\right)$ which cover the sphere as a differentiable manifold. All the points on the invariant sphere at infinity in the coordinates of any local charts $U_{i}$ and $V_{i}$ have $z_{3}=0$. The points in the interior of the Poincaré ball, which is diffeomorphic to $\mathbb{R}^{3}$, are given in the local charts $U_{i}$ by $z_{3}>0$ and in the local charts $V_{i}$ by $z_{3}<0$. See Fig. 14 for an illustration of the sphere $\mathbb{S}^{2}$ at infinity and the local charts $U_{i}$ and $V_{i}$ with their orientation. We use this compactification technique to study the phase portrait of system (1) near and at infinity and to prove Theorem 3.

The expression of system (1) in the local chart $U_{1}$ is $\dot{z}_{1}=-z_{1}^{2} z_{3}-z_{1} z_{2}-z_{3}$, 
Fig. 14 Local charts $U_{i}$, $i=1,2,3$, used to draw the phase portrait of the Sprott A system (1) on the sphere $\mathbb{S}^{2}$ at infinity. The charts $V_{i}$, $i=1,2,3$, are diametrically opposed to $U_{i}$

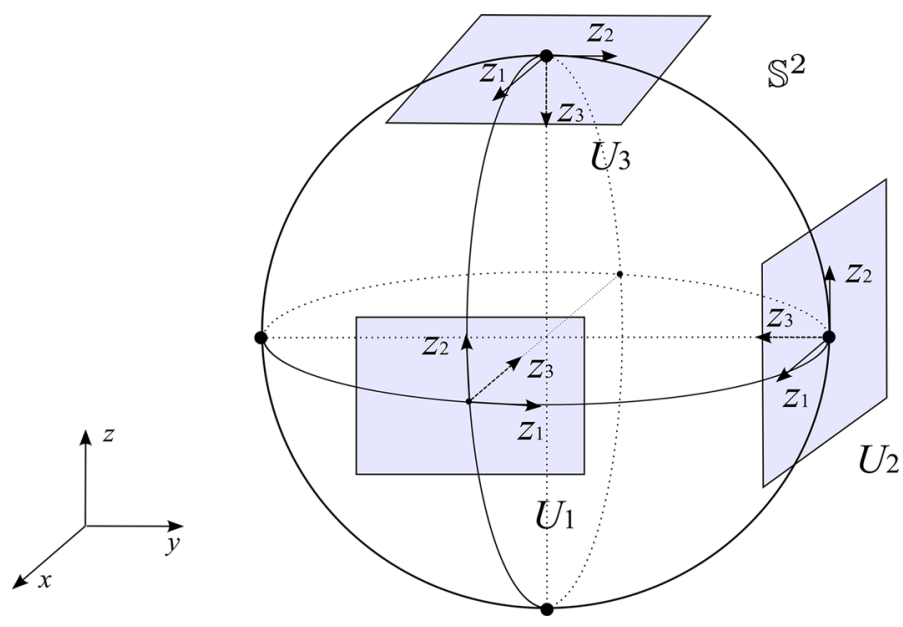

$\dot{z}_{3}=z_{3}\left(z_{1} z_{3}+z_{2}\right)$.

$\dot{z}_{3}=-z_{3}^{2} z_{1}$.

Consider system (5) restricted to the invariant plane $z_{3}=0$. The restricted system has no equilibrium points. Note that $\dot{z}_{1}>0$ for $z_{1}>0, z_{2}>0$ and $z_{1}<0$, $z_{2}<0 ; \dot{z}_{1}=0$ on the $z_{1}$ - and $z_{2}$-axis; and $\dot{z}_{1}<0$ for $z_{1}<0, z_{2}>0$ and $z_{1}>0, z_{2}<0$. Hence, the flow of the restricted system in the direction of $z_{1}$-axis is increasing in the quadrants I and III, constant on the $z_{1}$ - and $z_{2}$-axis and decreasing in the quadrants II and IV. Moreover, $\dot{z}_{2}>0$, then the flow is always increasing in the direction of $z_{2}$-axis. The phase portrait of system (5) restricted to the invariant plane $z_{3}=0$ is shown in Fig. 15b.

The flow in the local chart $V_{2}$ restricted to $z_{3}=0$ is the same as the flow in the local chart $U_{2}$ reversing appropriately the time direction.

The expression of system (1) in the local chart $U_{3}$ is given by

$$
\begin{aligned}
\dot{z}_{1}= & -z_{1} z_{2}^{2}+a z_{1} z_{3}^{2}+z_{2} z_{3}, \quad \dot{z}_{2}=-z_{2}^{3}+a z_{2} z_{3}^{2} \\
& -z_{1} z_{3}-z_{2}, \dot{z}_{3}=z_{3}\left(-z_{2}^{2}+a z_{3}^{2}\right) .
\end{aligned}
$$

Consider system (6) restricted to the invariant plane $z_{3}=0$. The $z_{1}$-axis is a line of equilibria of the restricted system, and the eigenvalues of the linear part of the system at these equilibrium points are -1 and 0 . Note that $\dot{z}_{1}>0$ for $z_{1}<0 ; \dot{z}_{1}=0$ on the $z_{2}$ axis; and $\dot{z}_{1}<0$ for $z_{1}>0$. Hence, the flow of the restricted system in the direction of $z_{1}$-axis is increasing for $z_{1}<0$, constant on the $z_{2}$-axis and decreasing for $z_{1}>0$. Moreover, $\dot{z}_{2}>0$ for $z_{2}<0$ and $\dot{z}_{2}<0$ for $z_{2}>0$. Then, the flow in the direction of $z_{2}$-axis is increasing for $z_{2}<0$ and decreasing for $z_{2}>0$, and the orbits tend toward the $z_{1}$-axis as $t \rightarrow+\infty$, which 


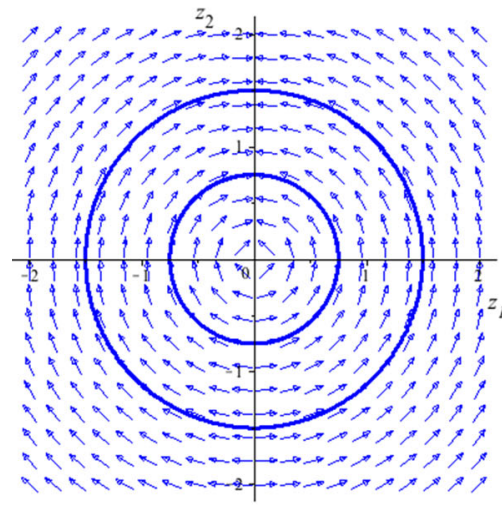

(a)

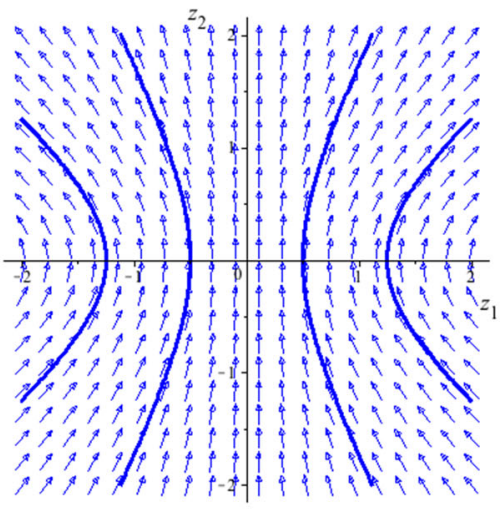

(b)

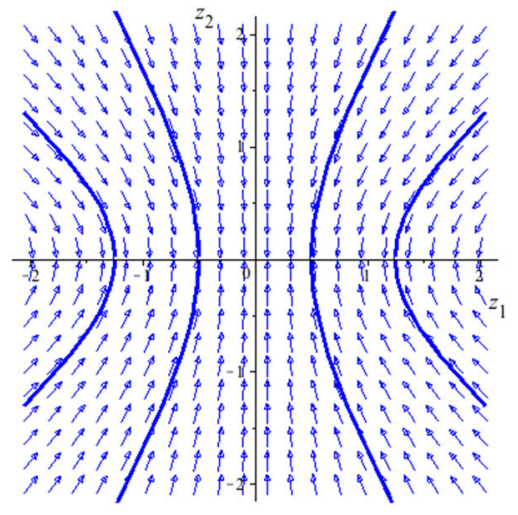

(c)

Fig. 15 Phase portraits in the local charts $U_{1}, U_{2}$ and $U_{3}$ restricted to the invariant plane $z_{3}=0$

Fig. 16 Flow of system (1) in an inside a neighborhood of the sphere $\mathbb{S}^{2}$ at infinity, in the local charts $U_{3}$ a and $V_{3} \mathbf{b}$

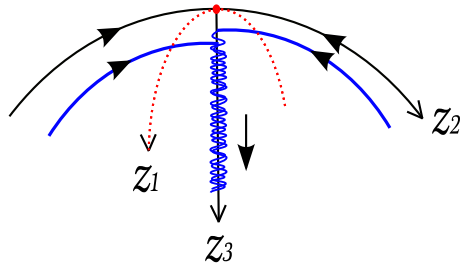

(a)

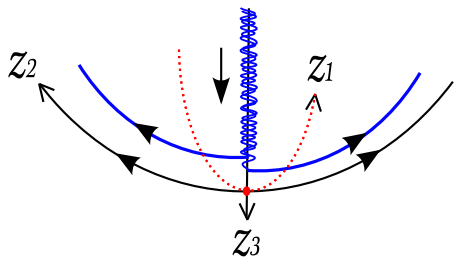

(b) is the line of equilibria. The phase portrait of system (6) restricted to the invariant plane $z_{3}=0$ is shown in Fig. 15c.

The flow in the local chart $V_{3}$ restricted to $z_{3}=0$ is the same as the flow in the local chart $U_{3}$ reversing appropriately the time direction.

Considering the analysis made in the local charts $U_{i}$ and $V_{i}, i=1,2,3$, restricted to the invariant plane $z_{3}=0$, we have a global picture of the dynamical behavior of system (1) on the sphere at infinity $\mathbb{S}^{2}$, which is given in Fig. 6 of Introduction. There exist a (great) circle of equilibria on the sphere at infinity and an infinite set of heteroclinic orbits connecting pairs of these equilibrium points. These heteroclinic orbits exist at infinity for all values of the parameter $a \in \mathbb{R}$, even when system (1) has chaotic behavior.

We claim that, for $a \neq 0$ small, the only orbit which escapes to infinity as $t \rightarrow \pm \infty$ is the one contained in the invariant $z$-axis and, consequently, there exist orbits homoclinic to the limit set near of the origin with large amplitude. Indeed, considering the flow of system (1) in the local chart $U_{3}$, given by system (6), the $z_{1}$-axis is a line of equilibria of system (6) and all orbits on the invariant plane $z_{3}=0$ tend to the equi- librium points of the $z_{1}$-axis, as shown in Fig. $15 \mathrm{c}$. Moreover, the $z_{3}$-axis (which corresponds to the $z$ axis in $\mathbb{R}^{3}$ ) is invariant by the flow of the compactified system at the local chart $U_{3}$ and the orbits with initial conditions in it go toward its positive direction (negative direction of the $z$-axis, see Fig. 14), because $\dot{z}_{3}=a z_{3}^{3}>0$, since we are considering $a>0$ and $z_{3}>0$ (which correspond to the points in the interior of the Poincaré ball, that is, in $\mathbb{R}^{3}$ ). Hence, from the numerical study developed in Sect. 3 and considering the continuity of the flow, the orbits in a neighborhood $z_{3}=\varepsilon>0$ of the invariant plane $z_{3}=0$ go toward the $z_{3}$-axis, and in a tubular neighborhood of it, they oscillate around this axis toward its positive direction, as drawn in Fig. 16a. The same is true in the local chart $V_{3}$ reversing appropriately the time direction (see Fig. 16b). Furthermore, there is no orbit, unless the one in the invariant $z_{3}$-axis, which tends to infinity, because of the persistence of the compact structure, described in Sect. 3.1. Therefore, the only orbit of system (1) which tends to infinity is the one in the invariant $z$-axis. Also, in a neighborhood of the sphere $\mathbb{S}^{2}$ at infinity, there exist orbits homoclinic to the limit set near the origin, which can be obtained connecting the orbits in the 


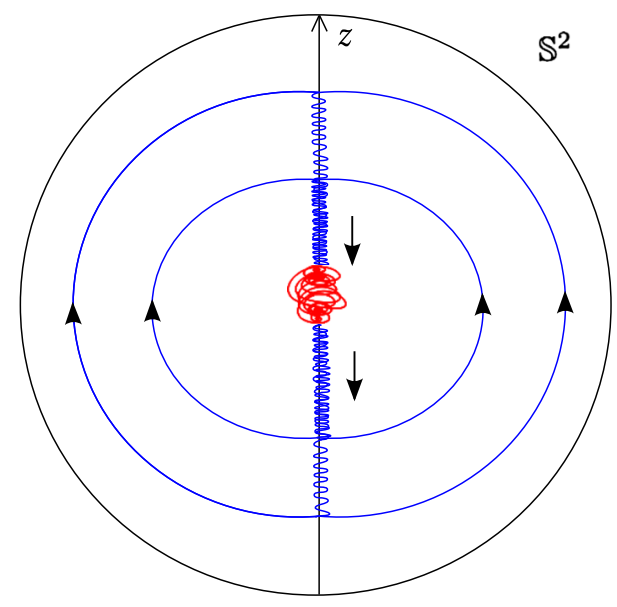

Fig. 17 Global dynamics of the Sprott A system (1) for $a>0$ small: far from the origin, almost all orbits are homoclinic to a limit set $(\mathrm{red})$, including the ones in a neighborhood of the sphere at infinity $\mathbb{S}^{2}$. (Color figure online)

local charts $U_{3}$ and $V_{3}$, over the deformed invariant sphere.

\section{Concluding remarks}

In this paper, we study the formation of hidden chaotic attractors and nested invariant tori in the Sprott A system (1), considering bifurcations obtained by varying the parameter $a$. For $a=0$, this system has a compact structure determined by invariant spheres, which are filled by heteroclinic orbits of south pole-north pole type. By a detailed numerical study of system (1) when $a>0$ small, we show that the heteroclinic orbits are destroyed and the spheres are no longer invariant algebraic surfaces of system (1). However, this compact structure although deformed is preserved, unless in a tubular neighborhood of the $z$-axis, and it occurs the formation of nested invariant tori in a neighborhood of the origin and a limit set encompassing them, which attracts almost all orbits of the Sprott A system in the future and in the past, as shown in the schematic Fig. 17. As the parameter value $a$ increases, the invariant tori and the limit set expand. Moreover, the limit set evolves into a hidden chaotic attractor for suitable choices of the parameter value $a$. This hidden chaotic attractor coexists with the nested invariant tori, also encompassing them, as shown in Fig. 13. From a global analysis of system (1), including its dynamics at infinity, we show that, for $a>0$ small, the only orbit which escapes to infinity is the one contained in the invariant $z$-axis, so we observe the existence of orbits with large amplitude (near the Poicaré sphere at infinity) which are homoclinic to the limit set or to a hidden chaotic attractor around the origin, as illustrated in Fig. 17. We can conclude that the bifurcation of the heteroclinic orbits contained on the invariant spheres, leading to the creation of a global homoclinic structure in the phase space, has a crucial role in the formation of nested invariant tori and hidden chaotic attractors. In [19], the author also studied the creation of chaotic attractors in the Lorenz system due to the bifurcation of orbits heteroclinic to a line of equilibria.

Finally, we observe that in [8], the authors gave the equations of seventeen differential systems, $\mathrm{NE}_{1}$ to $\mathrm{NE}_{17}$, having no equilibria, but presenting chaotic dynamics (the Sprott A system studied here corresponds to the case $\mathrm{NE}_{1}$ ). After a numerical study of these systems, we can say that the mechanism of formation of hidden attractors in systems $\mathrm{NE}_{6}, \mathrm{NE}_{8}$ and $\mathrm{NE}_{9}$ is similar to the one described here for Sprott A system. However, these systems do not present spheres (or other compact algebraic surfaces) as invariant algebraic surfaces, which makes their studies far more complicated. The other systems given in [8] have different mechanisms of formation of hidden attractors, which are being studied by us, and will be presented in forthcoming works.

Acknowledgements The first author is supported by FAPESP Process number 2013/24541-0, by CNPq Grant Number 308315/ 2012-0 and by CAPES Grant Number 88881.030454/2013 from the program CSF-PVE. The second author is supported by FAPESP Process Number 2013/26602-7.

\section{References}

1. Chen, G.R., Ueta, T.: Yet another chaotic attractor. Int. J. Bifurcat. Chaos 9, 1465-1466 (1999)

2. Cima, A., Llibre, J.: Bounded polynomial vector fields. Trans. Am. Math. Soc. 318, 557-579 (1990)

3. Danca, M.F.: Hidden transient chaotic attractors of Rabinovich-Fabrikant system. Nonlinear Dyn. 86, 12631270 (2016)

4. Dudkowski, D., Jafari, S., Kapitaniak, T., Kuznetsov, N., Leonov, G.A., Prasad, A.: Hidden attractors in dynamical systems. Phys. Rep. 637, 1-50 (2016)

5. Gotthans, T., Petržela, J.: New class of chaotic systems with circular equilibrium. Nonlinear Dyn. 73, 429-436 (2015)

6. Hoover, W.G.: Remark on 'Some simple chaotic flows'. Phys. Rev. E 51, 759-760 (1995) 
7. Jafari, S., Sprott, J.C.: Simple chaotic flows with a line equilibrium. Chaos Solitons Fractals 57, 79-84 (2013)

8. Jafari, S., Sprott, J.C., Golpayegani, S.M.R.H.: Elementary quadratic chaotic flows with no equilibria. Phys. Lett. A 377, 699-702 (2013)

9. Jafari, S., Sprott, J.C., Nazarimehr, F.: Recent new examples of hidden attractors. Eur. Phys. J. Special Top. 224, 14691476 (2015)

10. Kingni, S.T., Jafari, S., Simo, H., Woafo, P.: Threedimensional chaotic autonomous system with only one stable equilibrium: analysis, circuit design, parameter estimation, control, synchronization and its fractional-order form. Eur. Phys. J. Plus 129, 76 (2014)

11. Lao, S.K., Shekofteh, Y., Jafari, S., Sprott, J.C.: Cost function based on Gaussian mixture model for parameter estimation of a chaotic circuit with a hidden attractor. Int. J. Bifurcat. Chaos 24, 1450010 (11 pages) (2014)

12. Leonov, G.A., Kuznetsov, N.V.: Hidden attractors in dynamical systems: from hidden oscillations in HilbertKolmogorov, Aizerman and Kalman problems to hidden chaotic attractor in Chua circuits. Int. J. Bifurcat. Chaos 23, 1330002 (69 pages) (2013)

13. Leonov, G.A., Kuznetsov, N.V., Mokaev, T.N.: Homoclinic orbits, and self-excited and hidden attractors in a Lorenzlike system describing convective fluid motion. Eur. Phys. J. Special Top. 224, 1421-1458 (2015)

14. Li, C., Sprott, J.C.: Coexisting hidden attractors in a 4-D simplified Lorenz system. Int. J. Bifurcat. Chaos 24, 1450034 (12 pages) (2014)

15. Llibre, J., Messias, M.: Global dynamics of the Rikitake system. Phys. D 238, 241-252 (2009)

16. Llibre, J., Messias, M., da Silva, P.R.: Global dynamics in the Poincare ball of the Chen system having invariant algebraic surfaces. Int. J. Bifurcat. Chaos 22, 1250154 (17 pages) (2012)

17. Lorenz, E.N.: Deterministic nonperiodic flow. J. Atmos. Sci. 20, 130-141 (1963)

18. Lü, J.H., Chen, G.R.: A new chaotic attractor coined. Int. J. Bifurcat. Chaos 12, 659-661 (2002)

19. Messias, M.: Dynamics at infinity and the existence of singularly degenerate heteroclinic cycles in the Lorenz system. J. Phys. A Math. Theor. 42, 115101 (18 pages) (2009)

20. Molaie, M., Jafari, S., Sprott, J.C., Golpayegani, S.M.R.H.: Simple chaotic flows with one stable equilibrium. Int. J. Bifurcat. Chaos 23, 1350188 (7 pages) (2013)
21. Pham, V.T., Jafari, S., Vaidyanathan, S., Volos, C., Wang, X.: A novel memristive neural network with hidden attractors and its circuitry implementation. Sci. China Tech. Sci. 59, 1-6 (2016)

22. Pham, V.T., Volos, C., Jafari, S., Vaidyanathan, S., Kapitaniak, T., Wang, X.: A chaotic system with different families of hidden attractors. Int. J. Bifurcat. Chaos 8, 1650139 (9 pages) (2016)

23. Posch, H.A., Hoover, W.G., Vesely, F.J.: Canonical dynamics of the Nosé oscillator: stability, order, and chaos. Phys. Rev. A 33, 4253-4265 (1986)

24. Rössler, O.: An equation for continuous chaos. Phys. Lett. A 57, 397-398 (1976)

25. Sandri, M.: Numerical calculation of Lyapunov exponents. Math. J. 6, 79-84 (1996)

26. Shahzad, M., Pham, V.T., Ahmad, M.A., Jafari, S., Hadaeghi, F.: Synchronization and circuit design of a chaotic system with coexisting hidden attractors. Eur. Phys. J. Special Top. 224, 1637-1652 (2015)

27. Sparrow, C.: The Lorenz Equations: Bifurcations, Chaos and Strange Attractors. Springer, New York (1982)

28. Sprott, J.C.: Some simple chaotic flows. Phys. Rev. E 50, R647-R650 (1994)

29. Wang, Z., Cang, S., Ochola, E.O., Sun, Y.: A hyperchaotic system without equilibrium. Nonlinear Dyn. 69, 531-537 (2012)

30. Wang, X., Chen, G.: A chaotic system with only one stable equilibrium. Commun. Nonlinear Sci. Numer. Simul. 17, 1264-1272 (2012)

31. Wei, Z., Pehlivan, I.: Chaos, coexisting attractors, and circuit design of the generalized Sprott $\mathrm{C}$ system with only two stable equilibria. Optoelectron. Adv. Mater. Rapid Commun. 6, 742-745 (2012)

32. Wei, Z., Yang, Q.: Dynamical analysis of a new autonomous 3-D chaotic system only with stable equilibria. Nonlinear Anal. Real World Appl. 12, 106-118 (2011)

33. Wei, Z., Zhang, W.: Hidden hyperchaotic attractors in a modified Lorenz-Stenflo system with only one stable equilibrium. Int. J. Bifurcat. Chaos 24, 1450127 (14 pages) (2014)

34. Wei, Z., Zhang, W., Wang, Z., Yao, M.: Hidden attractors and dynamical behaviors in an extended Rikitake system. Int. J. Bifurcat. Chaos 25, 1550028 (11 pages) (2015) 